In Cres. Vol. $4 N^{\circ}$ 2: pp. 337-348, 2013

\title{
ESTILO DE VIDA Y FACTORES BIOSOCIOCULTURALES DEL ADULTO. SECTOR POCÓS - CASERÍO POCÓS - MORO, 2011*
}

\author{
LIFE STYLES AND BIOSOCIOCULTURAL FACTORS OF ADULTS. \\ POCÓS, VILLAGE POCÓS - MORO, 2011
}

\author{
Kenia Betzy Jaramillo Mampis ${ }^{1}$, \\ Elena Esther Reyna Márquez²
}

\section{RESUMEN}

La investigación tiene como objetivo general determinar la relación entre el estilo de vida y factores biosocioculturales del adulto, en el Sector Pocós - Caserío Pocós - Moro, 2011. La investigación fue cuantitativa-descriptivo correlacional, con un universo muestral de 64 adultos, a quienes se le aplicó la escala del estilo de vida y el cuestionario sobre factores biosocioculturales. Los datos fueron procesados con el Software SPSS versión 18.0. Para establecer la relación entre las variables de estudio se aplicó la prueba de independencia de criterios Chi cuadrado, con el $95 \%$ de confiabilidad y significancia de $p<0.05$. Se obtuvieron los siguientes resultados: La mayoría de los adultos presentan un estilo de vida no saludable y un porcentaje significativo, saludable. En relación a los factores biosocioculturales tenemos que la totalidad de adultos son de religión católica; la mayoría percibe ingresos de 100 a 599 soles mensuales; más de la mitad es de estado civil unión libre y casado; la mitad es adulto maduro y de sexo femenino y masculino; menos de la mitad es de grado de instrucción primaria completa y de ocupación ama de casa. No existe relación estadísticamente significativa entre el estilo de vida y los factores biosocioculturales: edad, sexo, grado de instrucción, religión, estado civil, ocupación e ingreso económico.

PALABRAS CLAVE: Estilo de vida, Factores biosocioculturales, Adultos.

* Recibido: 14 de junio del 2013; aprobado: 28 de noviembre del 2013.

1 Estudiante de Enfermería de la Universidad Católica Los Ángeles de Chimbote.

2 Licenciada en Enfermería, Docente tutora investigadora de la Escuela Profesional de Enfermería de la Universidad Católica Los Ángeles de Chimbote. 


\begin{abstract}
The overall research aims to determine the relationship between lifestyle and adult biosocioculturales factors. Pocós, Village Pocós, Moro, 2011. The research was quantitative-descriptive correlational with a sampling universe of 64 adults, who have applied the scale and lifestyle questionnaire biosocioculturales factors. The data were processed with SPSS software version 18.0. To establish the relationship between the study variables was applied to test the independence of criteria Chi square, with $95 \%$ reliability and significance of $p<0.05$. The following results were obtained: Most adults have an unhealthy lifestyle and a significant percentage, healthy. Regarding biosocioculturales factors we have: The adults are all Catholic. Most have income of 100-599 soles per month. More than half is marital cohabitation and married. Half is mature adult female and male. Less than half is complete primary education level and occupation housewife. There is no statistically significant relationship between lifestyle and biosocioculturales factors: age, sex, level of education, religion, marital status, occupation and income of adults Sector Hamlet few few-Moro.
\end{abstract}

KEY WORDS: Lifestyle factors biosocioculturales and adults.

\title{
I. INTRODUCCIÓN
}

La preservación de la salud física y mental constituye una responsabilidad individual, con independencia de las acciones dirigidas a su conservación en el ciudadano y representa un deber cívico para evitar añadir una carga económica o sentimental a corto o lejano plazo, a la familia o a la propia sociedad, dando la posibilidad de alcanzar una vejez sana, portadora de ricas y ejemplarizantes experiencias para los más jóvenes. Tal legado es un estilo de vida.

El estilo de vida puede ser entendido como los hábitos de vida, la forma de vida, conjunto de comportamientos o actitudes que desarrollan las personas que unas veces son saludables y otras, nocivas para la salud. ${ }^{1}$

La salud de las personas adultas se asocia al estilo de vida, debido a que el estilo de vida promotor de salud procura el bienestar del ser humano y los estilos de vida poco saludables se asocian a factores de riesgo que contribuyen a la presencia de las enfermedades. ${ }^{2}$

Los problemas de salud en las personas son un asunto que debe tomarse en cuenta desde la promoción y la prevención. Más aún si se considera el bajo acceso a los servicios de salud (seguridad social), programas especializados, el alto costo de los tratamientos y las irreparables pérdidas ocasionadas por enfermedad que son prevenibles. ${ }^{2}$ 
En los países subdesarrollados los estilos de vida poco saludables son los que causan la mayoría de enfermedades. El estilo de vida que tiene la persona en sus años previos si nunca hizo actividad física, su nutrición fue deficiente o tuvo hábitos nocivos, tendrá problemas en su vejez. De ahí la importancia de las actividades y programas de promoción y prevención en la salud. ${ }^{1}$

A esta realidad no escapa el adulto del Sector de Pocós - Caserío Pocós, que se ubica en la jurisdicción del Puesto de Salud de Pocós. Las principales actividades a las que se dedica la población adulta son: la agricultura, el comercio, la crianza de animales (patos, gallinas, gallos de lucha, pavos, cerdos, ovejas y cuyes). Además, las actividades recreativas y deportivas que realiza la población son el futbol y voley, que las realizan por las tardes después del trabajo. ${ }^{3}$

La presente investigación es importante para la profesión de enfermería. Es útil porque se pueden posibilitar programas estratégicos que promuevan un estilo de vida saludable en los usuarios. Así, desde una perspectiva educativa y preventiva, fortalecerá su rol de liderazgo dentro del equipo de salud.

\section{PROBLEMA}

¿Existe relación entre el estilo de vida y los factores biososcioculturales del adulto en el Sector Pocós - Caserío Pocós - Moro, 2011?

\section{OBJETIVO GENERAL}

Determinar la relación entre el estilo de vida y los factores biosocioculturales del adulto en el Sector Pocós- Caserío Pocós-Moro, 2011.

\section{OBJETIVOS ESPECÍFICOS}

1. Valorar el estilo de vida del adulto en el Sector Pocós - Caserío Pocós Moro.

2. Identificar los factores biosocioculturales: edad, sexo, grado de instrucción, religión, estado civil, ocupación e ingreso económico del adulto. Sector Pocós - Caserío Pocós - Moro.

\section{FUNDAMENTACIÓN}

La presente investigación se fundamenta en las bases conceptuales de estilo de vida y factores biosocioculturales de la persona. 
Según Lalonde, M. en Tejera, J. ${ }^{4}$, los estilos de vida serían la forma de vivir de las personas, que al igual que el medio ambiente, están determinados por un conjunto de factores (físicos, sociales o laborales) que dependerán, tanto del individuo como del complejo entorno que le rodea, dando lugar a estilos de vida más o menos saludables.

Según Bibeaw y Col. en Maya, L. ${ }^{5}$, desde una perspectiva integral, es necesario considerar el estilo de vida como parte de una dimensión colectiva y social, que comprende tres aspectos interrelacionados: el material, el social y el ideológico. En lo material, el estilo de vida se caracteriza por manifestaciones de la cultura material: vivienda, alimentación, vestido. En lo social, según las formas y estructuras organizativas: tipos de familia, grupos de parentesco, redes sociales de apoyo y sistemas de soporte como las instituciones y asociaciones. En el plano ideológico, los estilos de vida se expresan a través de las ideas, valores y creencias que determinan las respuestas o comportamientos a los distintos sucesos de la vida.

\section{HIPÓTESIS}

Existe relación significativa entre el estilo de vida y los factores biosocioculturales del adulto del Sector Pocós- Caserío- Pocós- Moro.

\section{METODOLOGÍA}

El presente estudio fue de tipo cuantitativo, prospectivo, de corte transversal. ${ }^{6,7}$ con diseño descriptivo-correlacional. ${ }^{8,9}$ La población estuvo conformada por 64 adultos, que residen en en el Sector Pocós - Caserío Pocós - Distrito de Moro del Departamento de Ancash, utilizando los siguientes criterios de inclusión: Adultos que residen más de 3 años en el Sector, adulto mayor de ambos sexos, adulto mayor que acepta participar en el estudio.

Para la recolección de datos se aplicó la técnica de la entrevista, utilizando como instrumentos: la escala del estilo de vida elaborado por Walker, Sechrist y Pender (modificado por la docente investigadora y tutoras de investigación, respectivamente; Delgado, R; Reyna, E y Díaz, R) y el cuestionario sobre factores biosocioculturales de la persona adulta, elaborado por Delgado, R; Reyna E y Díaz, R. en base a criterios previstos para la unidad de análisis en estudio, los cuales se garantizaron en su validez y confiabilidad, obteniendo en la validez un valor der $>0,20^{6,7}$ y la confiabilidad ${ }^{10}$ con un valor de 0.79 a través de la prueba alfa de cronbach. 


\section{RESULTADOS}

Tabla 01

ESTILO DE VIDA DEL ADULTO. SECTOR POCÓS CASERÍO POCÓS - MORO, 2011

\begin{tabular}{lcc}
\hline Estilo de vida & Frecuencia & Porcentaje \\
\hline Saludable & 20 & 31,3 \\
No saludable & 44 & 68,7 \\
\hline Total & 64 & 100,0 \\
\hline $\begin{array}{l}\text { Fuente: Escala de estilo de vida elaborada por: Walker, Sechrist y Pender, } \\
\text { modificado por: Díaz, R; Reyna, E; Delgado, R. Aplicado al adulto. Sector Pocós- }\end{array}$ \\
$\begin{array}{l}\text { Caserío Pocós- Moro, Diciembre 2011. }\end{array}$
\end{tabular}

Tabla 02

FACTORES BIOSOCIOCULTURALES DEL ADULTO.

SECTOR POCÓS - CASERÍO POCÓS - MORO, 2011

\begin{tabular}{lc}
\hline FACTORES BIOLÓGICOS & \\
\hline Sexo & N \\
Masculino & 32 \\
Femenino & 32 \\
Total & 64 \\
Edad & N \\
20 a 35 & 18 \\
36 a 59 & 32 \\
60 a más & 14 \\
Total & 64
\end{tabular}

\begin{tabular}{lr}
\hline FACTORES CULTURALES & \\
\hline Grado de instrucción & N \\
Analfabeto(a) & 9 \\
Primaria incompleta & 7 \\
Primaria completa & 22 \\
Secundaria incompleta & 17 \\
Secundaria completa & 7 \\
Superior incompleta & 1 \\
Superior completa & 1 \\
Total & 64 \\
Religión & N \\
Católico(a) & 64 \\
Total & 64 \\
\hline
\end{tabular}




\begin{tabular}{lr}
\hline FACTORES SOCIALES & \\
\hline Estado civil & N \\
Soltero(a) & 6 \\
Casado(a) & 19 \\
Viudo(a) & 1 \\
Unión libre & 36 \\
Separado(a) & 2 \\
Total & 64 \\
Ocupación & $\mathbf{N}$ \\
Obrero(a) & 5 \\
Empleado(a) & 3 \\
Ama de casa & 27 \\
Estudiante & 1 \\
Agricultor & 20 \\
Otras & 8 \\
Total & 64 \\
Ingreso económico (soles) & $\mathbf{N}$ \\
Menos de 100 & 0 \\
De 100 a 599 & 53 \\
De 600 a 1000 & 6 \\
Más de 1000 & 5 \\
Total & 64 \\
\hline Fuente: Cuestionario de factores biosocioculturales, elaborado por: Díaz, R; \\
Reyna, E; Delgado, R. Aplicado al adulto. Sector Pocós- Caserío Pocós- Moro, \\
Diciembre 2011. \\
\hline
\end{tabular}

Tabla 03

FACTORES BIOLÓGICOS Y ESTILO DE VIDA DEL ADULTO. SECTOR POCÓS - CASERÍO POCÓS - MORO, 2011

\begin{tabular}{|c|c|c|c|c|c|c|}
\hline \multirow{3}{*}{ Sexo } & \multicolumn{4}{|c|}{ Estilo de vida } & & \\
\hline & \multicolumn{2}{|c|}{ Saludable } & \multicolumn{2}{|c|}{ No saludable } & \multicolumn{2}{|c|}{ Total } \\
\hline & $\mathrm{n}$ & $\%$ & $\mathbf{n}$ & $\%$ & $\mathbf{n}$ & $\%$ \\
\hline Masculino & 11 & 17,2 & 21 & 32,8 & 32 & 50,0 \\
\hline Femenino & 9 & 14,1 & 23 & 35,9 & 32 & 50,0 \\
\hline Total & 20 & 31,3 & 44 & 68,8 & 64 & 100,0 \\
\hline
\end{tabular}

Prueba de Chi-cuadrado de independencia

$$
\begin{gathered}
\mathrm{X}^{2}=0,291 ; 1 \mathrm{gl} \\
\mathrm{P}=0,590>0,05
\end{gathered}
$$

No existe relación estadísticamente significativa. 
Tabla 03 (Cont.)

\begin{tabular}{|c|c|c|c|c|c|c|}
\hline \multirow{3}{*}{ Edad } & \multicolumn{4}{|c|}{ Estilo de vida } & & \\
\hline & \multicolumn{2}{|c|}{ Saludable } & \multicolumn{2}{|c|}{ No saludable } & \multicolumn{2}{|c|}{ Total } \\
\hline & $\mathrm{n}$ & $\%$ & $\mathbf{n}$ & $\%$ & $\mathbf{n}$ & $\%$ \\
\hline 20 a 35 & 6 & 9,4 & 12 & 18,8 & 18 & 28,1 \\
\hline 36 a 59 & 10 & 15,6 & 22 & 34,4 & 32 & 50,0 \\
\hline 60 a más & 4 & 6,3 & 10 & 15,6 & 14 & 21,9 \\
\hline Total & 20 & 31,3 & 44 & 68,8 & 64 & 100,0 \\
\hline
\end{tabular}

Prueba de Chi-cuadrado de independencia

$$
\begin{gathered}
\mathrm{X}^{2}=0,083 ; 2 \mathrm{gl} \\
\mathrm{P}=0,959>0,05
\end{gathered}
$$

No existe relación estadísticamente significativa.

Fuente: Cuestionario de factores biosocioculturales elaborado por: Diaz, R; Reyna, E; Delgado, R y escala de estilo de vida elaborada por: Walker, Sechrist y Pender, modificada por: Díaz, R; Reyna, E; Delgado, R. Aplicado al adulto, Sector Pocós- Caserío Pocós- Moro, Diciembre 2011.

\section{Tabla 04}

FACTORES CULTURALES Y ESTILO DE VIDA DEL ADULTO. SECTOR POCÓS -

\begin{tabular}{|c|c|c|c|c|c|c|}
\hline \multirow{3}{*}{$\begin{array}{l}\text { Grado de } \\
\text { instrucción }\end{array}$} & \multicolumn{4}{|c|}{ Estilo de vida } & & \\
\hline & \multicolumn{2}{|c|}{ Saludable } & \multicolumn{2}{|c|}{ No saludable } & \multicolumn{2}{|c|}{ Total } \\
\hline & $\mathrm{n}$ & $\%$ & $\mathbf{n}$ & $\%$ & $\mathbf{n}$ & $\%$ \\
\hline Analfabeto(a) & 0 & 0,0 & 9 & 14,1 & 9 & 14,1 \\
\hline Primaria incompleta & 3 & 4,7 & 4 & 6,3 & 7 & 10,9 \\
\hline Primaria completa & 8 & 12,5 & 14 & 21,9 & 22 & 34,4 \\
\hline Secundaria incompleta & 4 & 6,3 & 13 & 20,3 & 17 & 26,6 \\
\hline Secundaria completa & 4 & 6,3 & 3 & 4,7 & 7 & 10,9 \\
\hline Superior incompleta & 1 & 1,6 & 0 & 0 & 1 & 1,6 \\
\hline Superior completa & 0 & 0 & 1 & 1,6 & 1 & 1,6 \\
\hline Total & 20 & 31,3 & 44 & 68,8 & 64 & 100,0 \\
\hline
\end{tabular}
CASERÍO POCÓS - MORO, 2011

Prueba de Chi-cuadrado de independencia

$$
\begin{aligned}
& X^{2}=10,108 ; 6 \mathrm{gl} \\
& P=0,120>0,05
\end{aligned}
$$

No existe relación estadísticamente significativa.

Fuente: Cuestionario de factores biosocioculturales elaborado por: Diaz, R; Reyna, E; Delgado, R y escala de estilo de vida elaborada por: Walker, Sechrist y Pender, modificada por: Díaz, R; Reyna, E; Delgado, R. Aplicado al adulto. Sector Pocós- Caserío Pocós- Moro, Diciembre 2011. 
Tabla 05

FACTORES SOCIALES Y ESTILO DE VIDA DEL ADULTO.

SECTOR POCÓS - CASERÍO POCÓS - MORO, 2011

\begin{tabular}{|c|c|c|c|c|c|c|}
\hline \multirow{3}{*}{ Estado civil } & \multicolumn{4}{|c|}{ Estilo de vida } & & \\
\hline & \multicolumn{2}{|c|}{ Saludable } & \multicolumn{2}{|c|}{ No saludable } & \multicolumn{2}{|c|}{ Total } \\
\hline & $\mathrm{n}$ & $\%$ & $\mathbf{n}$ & $\%$ & $\mathbf{n}$ & $\%$ \\
\hline Soltero(a) & 0 & 0 & 6 & 9,4 & 6 & 9,4 \\
\hline Casado(a) & 6 & 9,4 & 13 & 20,3 & 19 & 29,7 \\
\hline Viudo(a) & 0 & 0 & 1 & 1,6 & 1 & 1,6 \\
\hline Unión libre & 12 & 3,1 & 24 & 37,5 & 36 & 56,3 \\
\hline Separado(a) & 2 & 3,1 & 0 & 0 & 2 & 3,1 \\
\hline Total & 20 & 31,1 & 44 & 68,8 & 64 & 100,0 \\
\hline
\end{tabular}

Prueba de Chi-cuadrado de independencia

$$
\begin{gathered}
\mathrm{X}^{2}=7,656 ; 4 \mathrm{gl} \\
\mathrm{P}=0,105>0,05
\end{gathered}
$$

\begin{tabular}{|c|c|c|c|c|c|c|}
\hline \multirow{3}{*}{ Ocupación } & \multicolumn{4}{|c|}{ Estilo de vida } & & \\
\hline & \multicolumn{2}{|c|}{ Saludable } & \multicolumn{2}{|c|}{ No saludable } & \multicolumn{2}{|c|}{ Total } \\
\hline & $\mathrm{n}$ & $\%$ & $\mathbf{n}$ & $\%$ & $\mathbf{n}$ & $\%$ \\
\hline Obrero(a) & 2 & 3,1 & 3 & 4,7 & 5 & 7,8 \\
\hline Empleado(a) & 1 & 1,6 & 2 & 3,1 & 3 & 4,7 \\
\hline Ama de casa & 9 & 14,1 & 18 & 28,1 & 27 & 42,2 \\
\hline Estudiante & 0 & 0 & 1 & 1,6 & 1 & 1,6 \\
\hline Agricultor & 7 & 10,9 & 13 & 20,3 & 20 & 31,2 \\
\hline Otros & 1 & 1,6 & 7 & 10,9 & 8 & 12,5 \\
\hline Total & 20 & 31,3 & 44 & 68,8 & 64 & 100,0 \\
\hline
\end{tabular}

No existe relación estadísticamente significativa.

Prueba de Chi-cuadrado de independencia

$$
\begin{gathered}
\mathrm{X}^{2}=0,793 ; 4 \mathrm{gl} \\
\mathrm{P}=0,939>0,05
\end{gathered}
$$

No existe relación estadísticamente significativa. 
Tabla 05 (Cont.)

\begin{tabular}{|c|c|c|c|c|c|c|}
\hline \multirow{3}{*}{$\begin{array}{l}\text { Ingresos } \\
\text { económicos } \\
\text { (soles) }\end{array}$} & \multicolumn{4}{|c|}{ Estilo de vida } & & \\
\hline & \multicolumn{2}{|c|}{ Saludable } & \multicolumn{2}{|c|}{ No saludable } & \multicolumn{2}{|c|}{ Total } \\
\hline & $\mathrm{n}$ & $\%$ & $\mathbf{n}$ & $\%$ & $\mathbf{n}$ & $\%$ \\
\hline Menos de 100 & 0 & 0 & 0 & 0 & 0 & 0 \\
\hline $100-599$ & 16 & 25 & 37 & 57,8 & 53 & 82,8 \\
\hline $600-1000$ & 3 & 4,7 & 3 & 4,7 & 6 & 9,4 \\
\hline Más de 1000 & 1 & 1,6 & 4 & 6,3 & 5 & 7,8 \\
\hline Total & 20 & 31,3 & 44 & 68,8 & 64 & 100,0 \\
\hline
\end{tabular}

Prueba de Chi-cuadrado de independencia

$$
\begin{gathered}
\mathrm{X}^{2}=2,423 ; 3 \mathrm{gl} \\
\mathrm{P}=0,489>0,05
\end{gathered}
$$

No existe relación estadísticamente significativa.

Fuente: Cuestionario de factores biosocioculturales elaborado por: Díaz, R; Reyna, E; Delgado R y escala de estilo de vida elaborada por: Walker, Sechrist y Pender, modificada por: Díaz, R; Reyna, E; Delgado R. Aplicado al adulto, Sector Pocós- Caserío Pocós- Moro, Diciembre 2011.

\section{DISCUSIÓN}

En la tabla 01 se muestra que en el Sector Pocos - Caserío Pocós - Moro, 2011 , el $31,3 \%$ (20) de adultos tiene estilo de vida saludable y un $68,7 \%$ (44) presenta un estilo de vida no saludable.

El estilo de vida es un constructo que se ha usado de manera genérica, como equivalente de la forma en que se entiende el modo de vivir, "estar en el mundo", expresado en los ámbitos del comportamiento, fundamentalmente en las costumbres. También está moldeado por la vivienda, el urbanismo, la posesión de bienes, la relación con el entorno y las relaciones interpersonales. ${ }^{11}$

Los estilos de vida saludables incluyen conductas de salud, patrones de conducta, creencias, conocimientos, hábitos y acciones de las personas para mantener, restablecer o mejorar su salud. Son producto de condicionantes personales, ambientales y sociales, que emergen no sólo del presente, sino también de la historia personal del sujeto. Se refieren a formas cotidianas de vivir que se aplican para las distintas esferas o áreas de funcionamiento del campo vital de una persona, satisfaciendo adecuadamente las propias necesidades y optimizando su calidad de vida. ${ }^{12}$

La mayoría de los adultos del Sector Pocós- Caserío Pocós, tiene estilo de 
vida no saludable. Los adultos del presente estudio en su mayoría consumen sus alimentos tres veces por día; sin embargo los alimentos ingeridos no son balanceados, consumen pocas verduras y frutas. Posiblemente ellos solo se preocupan por saciar su hambre y llenarse de energías para regresar al trabajo, el cual requiere de mucho esfuerzo físico, ya que existe un gran número de agricultores y en el caso de las amas de casa, ellas también ayudan en el trabajo, después de realizar sus quehaceres domésticos van al campo y ayudan en los trabajos que requieren menos esfuerzo físico.

Un estilo de vida poco saludable es causa de numerosas enfermedades, como la obesidad o el estrés. Comprende hábitos como el consumo de sustancias tóxicas (alcohol, drogas), el tabaquismo, el sedentarismo, las prisas, la exposición a contaminantes, etc. ${ }^{13}$

En la tabla 02 se reporta una distribución porcentual de los factores biosocioculturales del adulto del Sector Pocós. En el factor biológico se observa que el porcentaje de adultos y adultas son iguales: $50 \%$ (32). El 50\% (32) tiene una edad entre 36- 59 años. Con respecto a los factores culturales, el 34,4 (22) de adultos tiene primaria completa, en cuanto a la religión el 100\% (64) son católicos. En lo referente a los factores sociales, el 56,3\% (36) de estado civil unión libre; el 42,2\% (27) son amas de casa y otros $31,2 \%$ (20) son agricultores. El $82,8 \%$ (53) de la población tienen un ingreso económico de 100 a 599 nuevos soles.

En cuanto al factor edad en la presente investigación se evidencia que la mayoría de adultos son maduros, seguidos de adultos jóvenes; después, adultos mayores. Los adultos jóvenes y maduros del sexo masculino en su mayoría se dedican a la agricultura; algunos de los adultos mayores también, pero ellos realizan trabajos menos pesados y en algunos casos solo ayudan a sus hijos en lo que puedan.

En la variable grado de instrucción, lo que prevaleció fue primaria completa, posiblemente porque antiguamente los padres no enviaban a sus hijos a estudiar, ya que preferían llevarlos al campo a trabajar. También existe una cantidad significativa de adultos analfabetos que no tuvieron la oportunidad de estudiar. Esto se debería a que antiguamente las personas pensaban que los estudios no eran de gran provecho y lo mejor era aprender actividades que les ayudaran en el futuro, actividades que les iban a dar el sustento. A esto se suma que en el sector solo hay I.E. primaria y anteriormente no había movilidad para transportarse con facilidad hasta el pueblo, donde hay I.E. secundaria. 
En la variable religión todos los adultos profesan la religión católica. En la variable estado civil encontramos que más de la mitad tiene estado civil unión libre, que se evidencia especialmente en las parejas jóvenes. En la ocupación las más prevalentes son las amas de casa y agricultores; el ingreso económico se evidencia que más de la mitad tiene un ingreso de 100-599 nuevos soles mensuales.

Según los resultados obtenidos no existe relación estadísticamente significativa entre los factores biosocioculturales y el estilo de vida, lo que nos indica que no influyen en los estilos de vida. Las personas somos seres que razonamos y seres que decidimos qué conductas optar.

\section{CONCLUSIONES}

1. La mayoría de los adultos presentan un estilo de vida no saludable y un porcentaje significativo, saludable.

2. En relación a los factores biosocioculturales, la totalidad de adultos son de religión católica. La mayoría percibe ingresos de 100 a 599 soles mensuales y son de estado civil unión libre y casado. La mitad es adulto maduro y de sexo femenino y masculino. Menos de la mitad es de grado de instrucción primaria completa y de ocupación ama de casa.

3. No existe relación estadísticamente significativa entre el estilo de vida y los factores biosocioculturales: sexo, edad, estado civil, grado de instrucción, religión, ocupación e ingreso económico.

\section{REFERENCIAS BIBLIOGRÁFICAS}

1 SCIELO - Scientific Electronic Library Online. CONCYTEC - PERU, Calle del comercio 197 San Borja Lima - Perú [Serie en Internet] [Citado el 2011 Abril 18]. [Alrededor de 1 pantalla]. Disponible desde el URL: http:www.scielo.org.pe

2 MiNISTERIO DE SALUD "Lineamientos para la dirección general de las personas adultas mayores" 2005. [Serie en Internet] [Citado el 2011 Abril 18]. [Alrededor de 1 pantalla]. Disponible desde el URL: www.upch.edu.pe/vrinve/gerontologia/minsapdf/lineamientos.pdf2

3 Flores G. Censo Poblacional Caserío de Pocós - Sistema de Vigilancia Comunal. Puesto de Salud de Pocós. Sector de Pocós- Caserío Pocós. 2011.

4 TEJeRA J. Situación de un problema de salud por Marc Lalonde. [Monografía de internet]. [Citado 2010 Agosto 19]. [Alrededor de 2 pantallas]. Disponible en URL: http://www.monografias.com/ trabajos56/situacion-salud/situacion-salud.shtml

5 MAYA L. Los estilos de vida saludables: componente de la calidad de vida. [Documento en internet]. Colombia; 2001. [Citado 2010 Ago. 12]. [Alrededor de 3 pantallas]. Disponible en URL: http://www.funlibre.org/documentos/lemaya1.htm 
6 Polit D, Hungler B Investigación Científica en Ciencias de la Salud. Ed. Interamericana - Mc. Graw-Hill; 4ta ed. México 2000.

7 HeRnANDEZ R. Metodología de la Investigación. [Artículo en internet]. México: Editorial Mc Graw Hill; 2003. [Citado 2010 Agosto 15]. Disponible en URL: http://www.metabase.net/docs/ unibe/ 03624.html.

8 Schoenbach V. Diseños de estudio analítico. [Monografía en internet]. 2004. [Citado 2010 Agosto 18]. [Alrededor de 44 pantallas]. Disponible en URL: http://www.epidemiolog.net/es/ endesarrollo/DisenosDeEstudioAnaliticos.pdf.

9 Canales F y Alvarado E. Metodología de la Investigación. Edit. Limusa, 20a. Reimpresión, México. 2004.

10 CRISTOPHER M. Validez y confiabilidad del instrumento. [Biblioteca virtual]. España [Citado 2010 Setiembre 12]. [Alrededor de 1 pantalla]. Disponible en URL: http://www.eumed.net/libros/ 2007b/303/validez\%20y \%20confiabilidad\%20del\%20instrumento.htm

11 Guerreo L, León A. Estilo de vida y salud. Edt. Med. [Serie en Internet]. Venezuela. 2010. [Citado en 2012 Julio 17]. 14 (48). [Alrededor de 1 pantalla]. Disponible desde URL: http://www.saber.ula.ve/bitstream/123456789/32226/1/articulo1.pdf

12 DuRÁ M. Estilos de vida y conductas de riesgo de los adolescentes universitarios. Rev. Red. [Serie en Internet].España. 2010. [Citada en 2013 Mayo 28]. 2 (1): 680-695 [Alrededor de 1 pantalla]. Disponible desde URL: http://revistareduca.es/index.php/reduca-enfermeria/article/viewFile/ 203/225

13 Aguilar R. Diseño educativo actividad física. [Documento en Internet]. Chile. 2012. [Citada en 2013 Mayo 28]. [Alrededor de 1 pantalla]. Disponible desde URL: http://www.buenastareas.com/ ensayos/Dise\%C3\%B1o-Educativo-Actividad-Fisica/25950803.html 